\title{
Características do ambiente alimentar comunitário e do entorno das residências das famílias beneficiárias do Programa Bolsa Família
}

\author{
Characteristics of the community food environment and home \\ surroundings of households beneficiaries of the "Bolsa Família" \\ (family aid) program
}

\author{
Melissa Luciana de Araújo (https://orcid.org/0000-0002-9932-2135) 1 \\ Gláucia Bernardes Silva (https://orcid.org/0000-0002-2402-693X) ${ }^{1}$ \\ Luana Lara Rocha (https://orcid.org/0000-0002-5963-6033) ${ }^{2}$ \\ Taiane Gonçalves Novaes (https://orcid.org/0000-0002-9180-5490) ${ }^{3}$ \\ Cláudia Aparecida Marliére de Lima (https://orcid.org/0000-0003-1513-9868) ${ }^{1}$ \\ Larissa Loures Mendes (https://orcid.org/0000-0003-0776-6845) ${ }^{4}$ \\ Milene Cristine Pessoa (https://orcid.org/0000-0002-1053-5450) ${ }^{4}$
}

${ }^{1}$ Programa de Pós-

Graduação em Saúde e Nutrição, Escola de Nutrição, Universidade Federal de Ouro Preto. Campus Universitário s/n, Morro do Cruzeiro. 35400-000 Ouro Preto MG Brasil.melissaluciana20@ gmail.com

${ }^{2}$ Programa de PósGraduação em Saúde Pública, Faculdade de Medicina, Universidade Federal de Minas Gerais. Belo Horizonte MG Brasil. ${ }^{3}$ Programa de PósGradução em Ciência da Nutrição, Departamento de Saúde e Nutrição. Universidade Federal de Viçosa. Viçosa MG Brasil. ${ }^{4}$ Departamento de Nutrição, Escola de Enfermagem, Universidade Federal de Minas Gerais. Belo Horizonte MG Brasil.

\begin{abstract}
Knowing the food environment to which the households benefiting from the Bolsa Familia Program (PBF) are exposed is necessary to identify exposure to environments that favor worse food choices. This work aimed to characterize the community food environment of the urban area of the municipality of Ouro Preto (MG), Brazil, and the surroundings of the homes of households that are beneficiaries of the PBF. This ecological study was conducted with data from the Single Registry and the State Treasury Department Systems, both from 2014, and from the 2010 Census. Spatial analyses were conducted to evaluate the clusters of beneficiary households and establishments. The peripheral and with lower per capita income census tracts (CTs) had little or no establishment for food sales, and greater agglomeration of PBF beneficiary households closer to unhealthy establishments (median: 65.73 meters). There was a higher concentration of - mainly unhealthy - food sales establishments in the central CTs and higher per capita income (59.2\%). Given the above, it becomes important to dialogue with other nutrition-related public policies to reduce inequalities and improve physical access to healthy food sales establishments.
\end{abstract}

Key words Food and nutrition security, Government programs, Public health
Resumo Conhecer o ambiente alimentar a que as famílias beneficiárias do Programa Bolsa Família $(P B F)$ estão expostas é necessário para identificar a exposição a ambientes que favoreçam piores escolhas alimentares. O objetivo deste trabalho foi caracterizar o ambiente alimentar comunitário da zona urbana do município de Ouro Preto e do entorno das residências das famílias beneficiárias do PBF. Trata-se de um estudo ecológico, conduzido com dados do Sistema de Cadastro Único e da Secretaria Estadual da Fazenda, ambos de 2014, e do Censo 2010. Foram realizadas análises espaciais para avaliar as aglomerações de familias beneficiárias e de estabelecimentos. Os setores censitários (SC) periféricos e com menor renda per capita apresentaram pouco ou nenhum local de venda de alimentos, bem como maior aglomeração de famílias beneficiárias do $P B F$, que estão mais próximas a estabelecimentos considerados não saudáveis (mediana: 65,73 metros). Verifcou-se maior concentração de locais de venda de alimentos, sobretudo não saudáveis, nos SC de localização central e de maior renda per capita $(59,2 \%)$. Nesse caso, torna-se relevante o diálogo com outras políticas públicas interrelacionadas com a nutrição, visando reduzir as iniquidades e contribuir para melhorar o acesso fisico a estabelecimentos de venda de alimentos saudáveis.

Palavras-chave Segurança alimentar e nutricional, Programas governamentais, Saúde pública 


\section{Introdução}

A abordagem do ambiente alimentar comunitário está relacionada com a disponibilidade, o acesso, a proximidade e a distribuição espacial de estabelecimentos de venda de alimentos onde as escolhas alimentares são realizadas. A compreensão desse cenário é importante, por ele ser um dos determinantes do comportamento alimentar e apresentar relação intrínseca com o direito humano à alimentação adequada (DHAA $)^{1-5}$. A presença de estabelecimentos de comercialização de alimentos na vizinhança, assim como a acessibilidade, disponibilidade e preço dos gêneros alimentícios, podem ser fatores que influenciam o comportamento alimentar dos indivíduos, sobretudo daqueles desfavorecidos economicamente $^{6,7}$.

A escassez de opções de alimentos in natu$r a$ ou minimamente processados na vizinhança, bem como o custo elevado desses alimentos, é uma barreira para a adoção de hábitos alimentares saudáveis e para a garantia do DHAA. Isso causa o deslocamento dos moradores para locais distantes de suas residências para obtenção de alimentos in natura e minimamente processados a preços acessíveis, ou pode provocar a redução do consumo desses alimentos ${ }^{8-10}$.

O Programa Bolsa Família (PBF), implantado nacionalmente em 2004, foi elaborado com o intuito de apoiar as famílias mais vulneráveis e garantir o direito aos serviços sociais básicos, sendo um de seus objetivos a promoção da segurança alimentar e nutricional (SAN) das famílias beneficiárias ${ }^{11}$. Entende-se por SAN, segundo a Lei Orgânica de Segurança Alimentar e Nutricional - LOSAN"12, "a realização do direito de todos ao acesso regular e permanente a alimentos de qualidade, em quantidade suficiente, sem comprometer o acesso a outras necessidades essenciais, tendo como base práticas alimentares promotoras de saúde que respeitem a diversidade cultural e que sejam ambiental, cultural, econômica e socialmente sustentáveis".

Conhecer o ambiente alimentar ao qual as famílias beneficiárias do PBF estão expostas se faz necessário para identificar a exposição a ambientes que favoreçam piores escolhas alimentares, o que dará subsídio para incentivar maiores esforços, da sociedade civil e dos governantes, na alteração desse ambiente, a fim de aumentar a disponibilidade de alimentos saudáveis, que contribuem para melhorar as condições de saúde dessa população. No que diz respeito às famílias beneficiárias, há uma abundância de estudos que abordam a temática da $\mathrm{SAN}^{13-21}$, e há outros que avaliaram o consumo alimentar dessa população ${ }^{22-26}$, no entanto, não foi encontrado nenhum estudo que tenha avaliado o ambiente alimentar comunitário das famílias beneficiárias do programa.

Diante do exposto, a avaliação da disponibilidade dos estabelecimentos de comercialização de alimentos na vizinhança dos beneficiários do PBF é importante para a garantia da SAN e na elaboração de políticas públicas que tenham o objetivo de melhorar o acesso a alimentos in natura e minimamente processados. Dessa forma, o presente estudo teve como objetivo caracterizar o ambiente alimentar comunitário da zona urbana de Ouro Preto (MG) e do entorno das residências das famílias munícipes beneficiárias do Programa Bolsa Família.

\section{Método}

\section{Região de estudo}

O estudo possui delineamento ecológico, realizado com dados obtidos para Ouro Preto, Minas Gerais. O município tinha 70.281 habitantes, segundo o censo realizado pelo Instituto Brasileiro de Geografia e Estatistica (IBGE) ${ }^{27} \mathrm{em} 2010$, com densidade demográfica de $56,41 \mathrm{hab} / \mathrm{km}^{2} \mathrm{e}$ Índice de Desenvolvimento Humano Municipal (IDHM) de 0,741. A extensão territorial de Ouro Preto é de $1.245,865 \mathrm{~km}^{2}$, apresentando relevo com declives acentuados, ruas estreitas e tortuosas e pavimentação com pedras irregulares, com disponibilidade limitada do transporte público ${ }^{28}$.

\section{Fontes de dados e variáveis de estudo}

Este estudo foi realizado a partir de três diferentes fontes de dados: a Classificação Nacional de Atividades Econômicas (CNAE), para obtenção dos dados acerca dos estabelecimentos de venda de alimentos; O censo realizado em 2010 pelo IBGE, no qual foram coletadas as informações referentes à renda dos setores censitários; $\mathrm{e}$ o Sistema de Cadastro Único para Programas Sociais do Governo Federal, contendo informações sobre as famílias beneficiárias do Programa Bolsa Família.

Para descrever o ambiente alimentar comunitário de Ouro Preto e do entorno das residências das famílias beneficiárias do PBF, foram solicitadas à Secretaria Estadual da Fazenda as informações do endereço e da Classificação Nacional 
de Atividades Econômicas (CNAE) dos estabelecimentos de venda de alimentos cadastrados no ano de 2014 em Ouro Preto (MG). As coordenadas geográficas (latitude e longitude) dos estabelecimentos de venda de alimentos foram obtidas a partir dos endereços utilizando o software Google Earth Pro.

A CNAE é um instrumento de padronização nacional dos códigos de atividades econômicas e dos critérios de enquadramento utilizados pelos diversos órgãos da administração tributária do país ${ }^{29}$. A CNAE foi utilizada para classificar os estabelecimentos conforme a atividade fim cadastrada, sendo proposta uma agregação com base na extensão e no propósito do-processamento dos alimentos que são predominantemente comercializados, considerando a classificação $\mathrm{NOVA}^{30}$, que agrupa os alimentos em in natura, minimamente processados, processados e ultraprocessados.

Com base na classificação da CAISAN ${ }^{31}$, os estabelecimentos foram agregados em quatro categorias:

Estabelecimentos saudáveis (hortifrutigranjeiros, açougues, peixarias, vendas de laticínios e frios, lojas de produtos naturais e feiras livres);

Estabelecimentos não saudáveis (lojas de conveniência, mercearias, bares, bomboniéres, lanchonetes e sorveterias);

Estabelecimentos mistos (padarias e restaurantes);

Hipermercados e supermercados: essa categoria foi analisada isoladamente devido à ausência de consenso na literatura sobre a real influência desses estabelecimentos no consumo alimentar dos indivíduos, considerando a ampla gama de alimentos disponibilizados nesses espa$\operatorname{ços}^{32,33}$.

Foram realizadas análises descritivas da distribuição dos estabelecimentos de venda de alimentos, agrupadas segundo as categorias descritas acima por setor censitário. O setor censitário foi a unidade geográfica utilizada para a análise do ambiente alimentar no entorno das residências das famílias beneficiárias do PBF. As informações sobre os setores censitários, a renda mensal dos domicílios e a renda média mensal per capita dos setores foram obtidas por meio do Censo $2010^{34}$

Optou-se por utilizar as informações de renda do censo de 2010 por serem as informações mais atuais no momento do estudo. A renda per capita dos setores foi categorizada em tercis, a fim de avaliar a distribuição das categorias dos estabelecimentos que comercializam alimentos conforme a condição socioeconômica da vizinhança.
A informação de renda foi utilizada para a avaliação da condição socioeconômica de cada setor. A densidade de famílias beneficiárias do PBF, a densidade de estabelecimentos de venda de alimentos agrupados por categoria e a renda média mensal per capita foram analisadas por setor censitário para a Sede do Município de Ouro Preto, totalizando 62 setores incluídos.

Por meio da Secretaria de Desenvolvimento Social, Habitação e Cidadania, foram coletadas as informações sobre as famílias beneficiárias do Programa Bolsa Família. Os dados foram obtidos no Sistema de Cadastro Único (CadÚnico) para Programas Sociais do Governo Federal.

Foram encontradas 1.403 famílias beneficiárias que residiam na zona urbana de Ouro Preto, correspondendo a 48,21\% do total de 2.910 famílias beneficiárias do PBF cadastradas de 2006 até dezembro de 2014 em todo o município (zonas urbana e rural). As coordenadas geográficas das residências dos beneficiários foram obtidas por meio do CadÚnico, sendo excluídas 24 famílias que possuíam informações incorretas sobre latitude e longitude, totalizando 1.379 famílias incluídas neste estudo.

\section{Análise dos dados}

A análise descritiva dos estabelecimentos de venda de alimentos contemplou o cálculo de medidas de tendência central (média, mediana e percentil 50) e de dispersão (desvio padrão, intervalo interquartil, mínimo e máximo). Para verificar a distribuição das categorias de estabelecimentos conforme a condição socioeconômica da vizinhança, foram utilizados dados de renda média mensal per capita de cada setor censitário. Depois a variável quantitativa contínua "renda per capita dos setores censitários" foi categorizada em tercis.

Adicionalmente, foi realizada a estimativa de densidade de Kernel, para visualizar as possíveis aglomerações de cada categoria de estabelecimentos de venda de alimentos, e a função $\mathrm{K}$ de Ripley univariada, para verificar a significância dessas aglomerações. Foi estimada a distância euclidiana (em linha reta) entre os estabelecimentos de venda de alimentos e as residências das famílias beneficiárias do PBF.

As análises espaciais foram realizadas por meio do software QGIS 2.14.4, adotando o datum SIRGAS 2000 (Sistema de Referência Geocêntrico para as Américas), visto que se trata do Sistema Geodésico Brasileiro ${ }^{35}$. E as análises descritivas foram realizadas no software SPSS 20.0. 


\section{Resultados}

Foram identificados 732 estabelecimentos de venda de alimentos, sendo $57(7,8 \%)$ categorizados como estabelecimentos saudáveis, 217 $(29,6 \%)$ como estabelecimentos mistos, 447 $(61,1 \%)$ estabelecimentos não saudáveis e 11 (1,5\%) como hipermercados/supermercados. A mediana de renda per capita mensal dos setores censitários foi de $\mathrm{R} \$ 586,00$ ( $\mathrm{R} \$ 264,00-\mathrm{R} \$$ $2.833,00)$ e a mediana da densidade de famílias beneficiárias do PBF por setor foi de 89,41 (0,0551,72) famílias (Tabela 1).

A mediana da densidade de todos os estabelecimentos de venda de alimentos por setor censitário foi de 15,19 (0,0-175,68) estabelecimentos. Para as categorias de estabelecimentos mistos e estabelecimentos não saudáveis, as medianas foram maiores $(3,94$ [0,0-58,56] e 9,13 [0,0-108,11] estabelecimentos, respectivamente), enquanto para as categorias de estabelecimentos saudáveis e hipermercados/supermercados foram nulas (Tabela 1). A mediana da distância euclidiana entre os estabelecimentos de venda de alimentos e a residência das famílias beneficiárias do $\mathrm{PBF}$ foi maior para os hipermercados/supermercados
532,96 metros $(12,38-2.624,92)$ e menor para os estabelecimentos de venda de alimentos não saudáveis 65,73 metros (0,23-1.263,42) (Tabela 1).

Ao avaliar a densidade dos estabelecimentos (por 1.000 habitantes) de acordo com os tercis de renda per capita do setor censitário, é possível observar que, conforme a renda aumenta, a quantidade de todos os tipos de estabelecimentos também cresce, sendo essa densidade maior para os estabelecimentos não saudáveis em todos os tercis (Tabela 2). Em relação à densidade total de estabelecimentos, $16,1 \%$ desses estão aglomerados no $1^{\circ}$ tercil de renda e $61,4 \%$ estão no $3^{\circ}$ tercil (Tabela 2).

Ao realizar as estimativas de densidade de Kernel (Figura 1), foi possível observar que, em regiões periféricas e com baixa renda per capita, há uma menor quantidade de todos os tipos de estabelecimentos de venda de alimentos, principalmente os saudáveis e hipermercados/supermercados. Por outro lado, as regiões centrais e de maior renda per capita têm uma maior quantidade de todos os tipos de estabelecimentos de venda de alimentos, sobretudo aqueles categorizados como não saudáveis (Figura 1). A densidade de Kernel também foi utilizada para visualizar a

Tabela 1. Descrição das variáveis por setor censitário e distância euclidiana entre os estabelecimentos de vendas de alimentos e a residência das famílias beneficiárias do Programa Bolsa Família de Ouro Preto, MG, 2014.

\begin{tabular}{|c|c|c|c|c|c|c|}
\hline & Mínimo & IIQ & Média & Mediana & DP & Máximo \\
\hline $\begin{array}{l}\text { Renda per capita do setor } \\
\text { censitário (R } \$ / \text { mês }\end{array}$ & 264,00 & $437,25-930,00$ & 755,81 & 586,00 & 463,92 & $2.833,00$ \\
\hline $\begin{array}{l}\text { Densidade de famílias beneficiárias } \\
\text { do } \mathrm{PBF} / \text { setor censitário }\end{array}$ & 0,00 & $46,66-158,92$ & 113,24 & 89,41 & 95,24 & 551,72 \\
\hline $\begin{array}{l}\text { Densidade de estabelecimentos } \\
\text { saudáveis/setor censitário }\end{array}$ & 0,00 & $0,00-2,32$ & 1,47 & 0,00 & 2,08 & 9,60 \\
\hline $\begin{array}{l}\text { Densidade de estabelecimentos } \\
\text { mistos/setor censitário }\end{array}$ & 0,00 & $1,73-6,58$ & 6,83 & 3,94 & 10,89 & 58,56 \\
\hline $\begin{array}{l}\text { Densidade de estabelecimentos } \\
\text { não saudáveis/setor censitário }\end{array}$ & 0,00 & $4,37-13,77$ & 12,87 & 9,13 & 15,34 & 108,11 \\
\hline $\begin{array}{l}\text { Densidade de hipermercados e } \\
\text { supermercados/setor censitário }\end{array}$ & 0,00 & $0,00-0,00$ & 0,41 & 0,00 & 1,22 & 6,21 \\
\hline $\begin{array}{l}\text { Densidade do total de } \\
\text { estabelecimentos/setor censitário }\end{array}$ & 0,00 & $8,21-21,95$ & 21,58 & 15,19 & 26,81 & 175,68 \\
\hline \multicolumn{7}{|l|}{ Distância euclidiana } \\
\hline Estabelecimentos saudáveis & 0,71 & $22,81-96,61$ & 355,59 & 181,18 & 385,18 & $1.885,36$ \\
\hline Estabelecimentos mistos & 0,51 & $101,91-480,03$ & 157,19 & 102,54 & 162,97 & $1.264,27$ \\
\hline Estabelecimentos não saudáveis & 0,23 & $53,20-204,24$ & 100,64 & 65,73 & 123,03 & $1.263,42$ \\
\hline Hipermercados/supermercados & 12,38 & $30,34-120,00$ & 605,14 & 532,94 & 379,11 & $2.624,92$ \\
\hline $\begin{array}{l}\text { Todas as categorias de } \\
\text { estabelecimentos }\end{array}$ & 0,23 & $350,68-796,09$ & 78,42 & 50,63 & 109,08 & $1.263,42$ \\
\hline
\end{tabular}


distribuição das famílias beneficiárias do PBF de acordo com os tercis de renda per capita do setor censitário. Das 1.379 famílias incluídas no estudo, 799 (57,9\%) residem em setores censitários categorizados no $1^{\circ}$ tercil de renda, $427(30,9 \%)$ no $2^{\circ}$ tercil e $153(11,1 \%)$ no $3^{\circ}$ tercil (Figura 1$)$.
A função K de Ripley univariada (Figura 2) indicou que, com exceção dos hipermercados/ supermercados, há aglomerações significativas de estabelecimentos de venda de alimentos em setores censitários localizados na sede da cidade de Ouro Preto (MG). E ao avaliar a densidade

Tabela 2. Distribuição dos estabelecimentos de venda de alimentos por 1.000 habitantes segundo tercis de renda per capita dos setores censitários. Ouro Preto, MG, 2014.

\begin{tabular}{lrrrr}
\hline \multirow{2}{*}{ Estabelecimentos } & \multicolumn{1}{c}{$\mathbf{1}^{\mathbf{0}}$ tercil } & \multicolumn{1}{c}{$\mathbf{2}^{\mathbf{o}}$ tercil } & \multicolumn{1}{c}{$\mathbf{3}^{\mathbf{0}}$ tercil } & \multicolumn{1}{c}{ Total } \\
\cline { 2 - 5 } & $\mathbf{n}(\mathbf{\%})$ & $\mathbf{n}(\mathbf{\%})$ & $\mathbf{n}(\%)$ & \multicolumn{1}{c}{$\mathbf{n}(\mathbf{\%})$} \\
\hline Saudáveis/1.000 habitantes & $20,5(22,6)$ & $31,0(34,1)$ & $39,4(43,3)$ & $90,9(100,0)$ \\
Mistos/1.000 habitantes & $45,7(10,8)$ & $91,9(21,7)$ & $285,8(67,5)$ & $423,4(100,0)$ \\
Não saudáveis/1.000 habitantes & $149,8(18,8)$ & $175,4(22,0)$ & $472,9(59,2)$ & $798,1(100,0)$ \\
Hipermercados e supermercados/1.000 & $0,0(0,0)$ & $2,4(9,4)$ & $23,1(90,6)$ & $25,5(100,0)$ \\
habitantes & & & & \\
Todas as categorias de & $216,0(16,1)$ & $300,7(22,5)$ & $821,2(61,4)$ & $1.337,9(100,0)$ \\
estabelecimentos/1.000 habitantes & & & & \\
\hline
\end{tabular}

Fonte: Autoras.

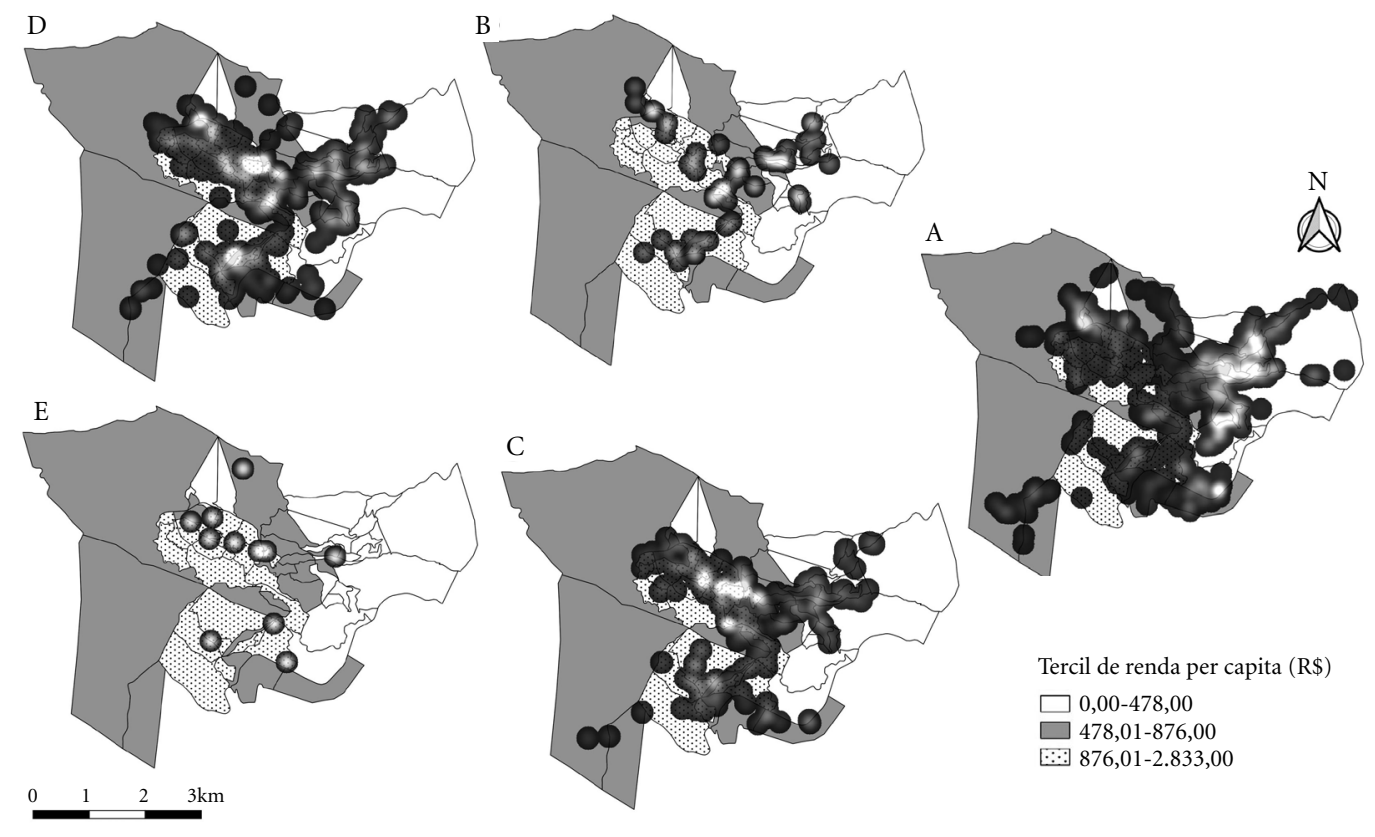

Figura 1. Estimativa de densidade de Kernel das categorias de estabelecimentos de venda de alimentos e das famílias beneficiárias do Programa Bolsa Família por tercis de renda per capita de Ouro Preto, MG, 2014.

Legenda: A - aglomeração das famílias beneficiárias do Programa Bolsa Família; B - aglomeração dos estabelecimentos saudáveis; C - aglomeração dos estabelecimentos mistos; D - aglomeração dos estabelecimentos não saudáveis; E - aglomeração de supermercados e hipermercados. 
dos estabelecimentos (por 1.000 habitantes) de acordo com os tercis de densidade de famílias beneficiárias do PBF, é possível observar que, à medida que a densidade de famílias aumenta, a densidade de todos os tipos de estabelecimentos diminui (Tabela 3).

\section{Discussão}

Os estabelecimentos que comercializam alimentos se concentravam nas regiões centrais e com maior renda da cidade, com uma maior quantidade de estabelecimentos categorizados como não saudáveis e mistos. Além disso, nas regiões
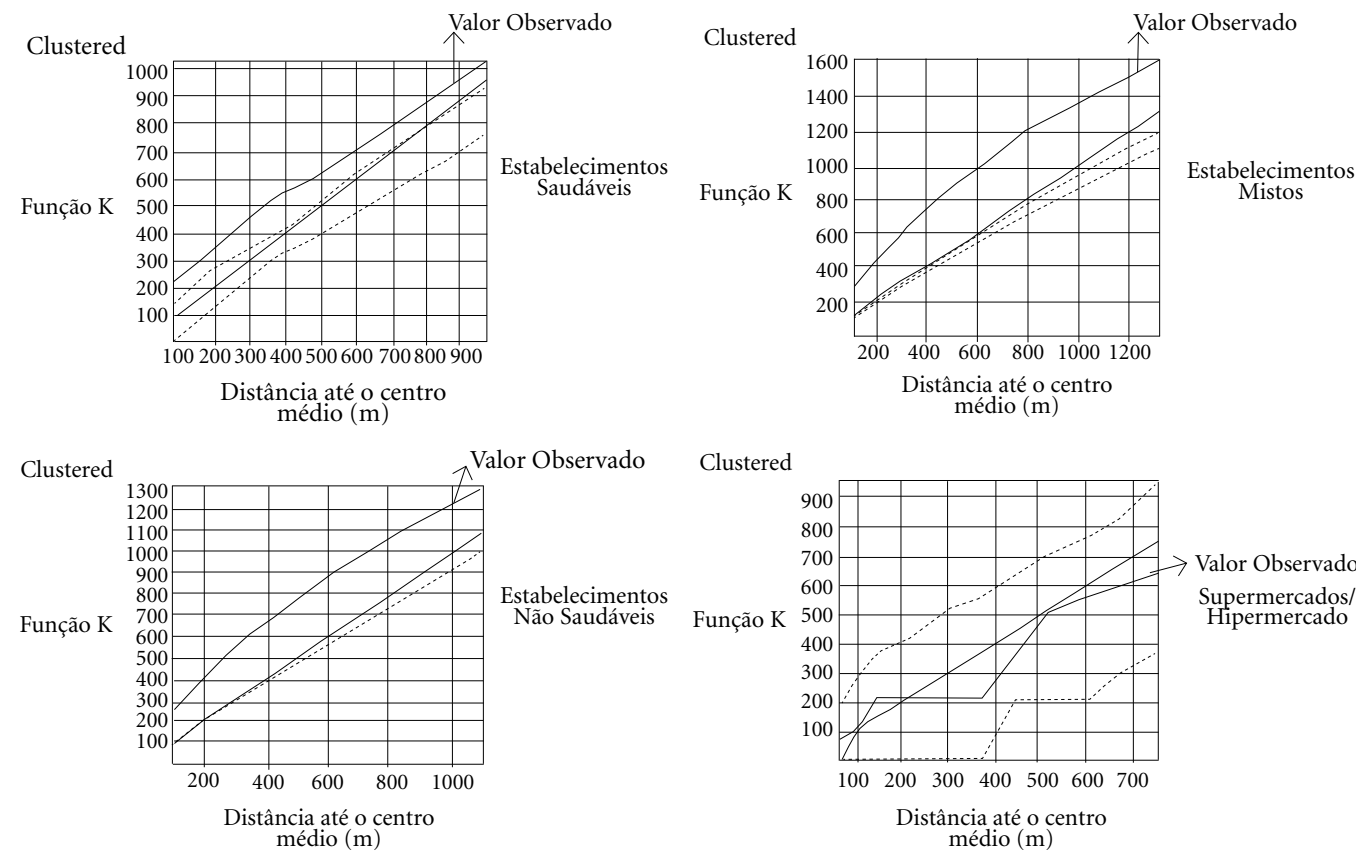

Clustered
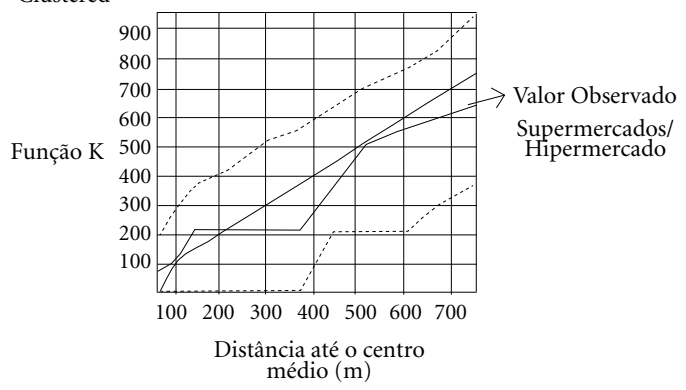

- Valor esperado
-... Valor observado
Envelope de confiança

Figura 2. Gráfico da função K de Ripley univariada para análise da distribuição espacial das categorias dos estabelecimentos de venda de alimentos.

Fonte: Autoras.

Tabela 3. Distribuição dos estabelecimentos de venda de alimentos por 1.000 habitantes segundo tercis de densidade de famílias beneficiárias do Programa Bolsa Família no setor censitário. Ouro Preto, MG, 2014.

\begin{tabular}{|c|c|c|c|c|}
\hline Estabelecimentos & $1^{\circ}$ tercil & $2^{\circ}$ tercil & $3^{\circ}$ tercil & Total \\
\hline & n (\%) & n (\%) & n (\%) & n (\%) \\
\hline Saudáveis/1.000 habitantes & $40,1(44,1)$ & $27,7(30,4)$ & $23,2(25,5)$ & $91,0(100,0)$ \\
\hline Mistos/1.000 habitantes & $277,2(65,5)$ & $91,4(21,6)$ & $54,8(12,9)$ & $423,4(100,0)$ \\
\hline Não saudáveis/1.000 habitantes & $450,7(56,5)$ & $207,0(25,9)$ & $140,4(17,6)$ & $798,1(100,0)$ \\
\hline $\begin{array}{l}\text { Hipermercados e supermercados/1.000 } \\
\text { habitantes }\end{array}$ & $18,4(72,2)$ & $6,0(23,5)$ & $1,1(4,3)$ & $25,5(100,0)$ \\
\hline $\begin{array}{l}\text { Todas as categorias de } \\
\text { estabelecimentos/ } 1.000 \text { habitantes }\end{array}$ & $786,3(58,8)$ & $332,0(24,8)$ & $219,6(16,4)$ & $1.337,9(100,0)$ \\
\hline
\end{tabular}

Fonte: Autoras. 
em que há uma maior concentração de famílias beneficiárias do PBF, há uma menor quantidade de todos os tipos de estabelecimentos de venda de alimentos, assim como uma menor distância até os estabelecimentos categorizados como não saudáveis.

A aglomeração de estabelecimentos de venda de alimentos ocorreu na região central da cidade e em regiões com maior renda per capita, sendo que a aglomeração de estabelecimentos não saudáveis foi 12 vezes maior do que a de estabelecimentos saudáveis, seguindo o mesmo padrão encontrado em cidades de grande porte ${ }^{36}$. Um estudo realizado na capital de São Paulo encontrou que as áreas com menor privação socioeconômica tinham maior densidade de todos os tipos de estabelecimentos de venda de alimentos ${ }^{37}$. Essa maior aglomeração de estabelecimentos classificados como não saudáveis pode induzir o consumo de alimentos com baixo valor nutricional, como os alimentos ultraprocessados. Pois além do acesso limitado a alimentos saudáveis, há a tendência de redução do preço dos alimentos ultraprocessados no Brasil, o que diminui o consumo de alimentos in natura ou minimamente processados $^{38-41}$.

Em contrapartida, nas regiões periféricas da cidade, em que a renda per capita é menor, há uma menor quantidade de todos os tipos de estabelecimentos que comercializam alimentos, chegando a não existir nenhum estabelecimento em alguns setores censitários, o que pode ser um dificultador no acesso físico ao alimento. Essa característica do ambiente comunitário pode ser um indicativo da existência de desertos alimentares, por serem regiões de menor renda e com baixa ou nenhuma disponibilidade de estabelecimentos que vendem alimentos saudáveis ${ }^{42}$.

Dados de estudo realizado em Florianópolis (SC) mostraram que as regiões de menor renda possuíam menor densidade de estabelecimentos de venda de alimentos saudáveis ${ }^{43}$. Além disso, outros estudos detectaram haver, nas regiões menos favorecidas economicamente, maior presença de estabelecimentos com menor variedade de alimentos, menor disponibilidade de comidas saudáveis, maior custo e/ou pior qualidade ${ }^{44-48}$.

Ouro Preto também apresenta esse problema de acesso físico aos estabelecimentos de venda de alimentos. Em estudo realizado com a população geral sobre os hábitos de compra de alimentos, foram apontados como principais problemas o alto preço e o número insuficiente de estabelecimentos que os vendam nos bairros ${ }^{49}$. Em relação aos tipos de estabelecimentos, os mais frequenta- dos foram os açougues, os supermercados, as padarias e as quitandas ${ }^{49}$. Além disso, a maior parte do gasto mensal era realizada nos supermercados, e os menores em feiras, varejões/sacolões, armazéns e vendedores ambulantes ${ }^{49}$.

Em relação às famílias beneficiárias, de acordo com relatório do IBASE $(2008)^{50}$, o dinheiro do PBF era gasto principalmente com alimentação, e quanto mais pobre era a família, maior a proporção da renda gasta com alimentação. Além disso, os alimentos de maior densidade calórica e menor valor nutritivo foram os mais consumidos $^{50}$. O consumo de alimentos com alta densidade calórica e baixo valor nutricional pela população em geral estão comumente relacionados a excesso de peso, obesidade e outras doenças crônicas não transmissíveis ${ }^{22}$.

Tal condição reforça a necessidade da intersetorialidade, como previsto na $\operatorname{LOSAN}^{12}$, que prevê o diálogo das políticas de assistência social com as políticas de saúde, planejamento urbano e agricultura, entre outras. Ter o acesso físico aos alimentos saudáveis comprometido pode reforçar a ineficiência do $\mathrm{PBF}$ em relação à violação do DHAA e às dimensões da $\mathrm{SAN}^{51-54}$.

O exercício da intersetorialidade nas ações, envolvendo sociedade civil, controle social e gestores de diferentes setores, como saúde, planejamento urbano e assistência social, bem como a agricultura e a SAN, pode contribuir para a implementação de ações de proteção alimentar, como os restaurantes populares, e de programas voltados para o abastecimento e a regulação de mercado, como as feiras-livres, as feiras da agricultura familiar e os sacolões municipais que oferecem folhosos e/ou legumes a preços acessíveis, ampliando o acesso aos alimentos saudáveis ${ }^{55}$

Quanto à proximidade dos estabelecimentos e sua influência no padrão alimentar da família, a literatura se mostra controversa. Entretanto, estudos recentes encontraram associações positivas entre a proximidade de supermercados e estabelecimentos de venda de alimentos saudáveis e o padrão de alimentação e estado nutricional das famílias ${ }^{28,36,37,56}$. Contudo, o presente estudo encontrou que os estabelecimentos de venda de alimentos não saudáveis estão mais próximos das residências das famílias beneficiárias do $\mathrm{PBF}$ podendo influenciar na escolha dos alimentos por conta da conveniência de ser um local mais próximo. Pesquisadores têm sugerido que indivíduos que têm acesso limitado ao transporte público ou privado podem ser mais vulneráveis ao impacto negativo de viver em um local onde há, majoritariamente, estabelecimentos não sau- 
dáveis. Para essas pessoas, tal proximidade é um fator determinante na redução dos custos associados à aquisição de alimentos ${ }^{57}$.

A proximidade entre estabelecimentos e residências é um elemento importante a ser considerado em Ouro Preto. Destaca-se a topografia da cidade, caracterizada por declives acentuados, $o$ que pode ser um problema para o acesso a alimentos, principalmente aqueles considerados saudáveis, cujos estabelecimentos estão localizados, em média, a maior distância dos domicílios ${ }^{42}$. Além disso, o município apresenta obstáculos físicos que interferem nas condições de mobilidade e de acessibilidade da população, com ruas estreitas e tortuosas, com pavimento em pedras irregulares e de pouca aderência, calçadas estreitas e malconservadas, rampas com inclinações excessivas, obstáculos intercalados e escadarias sem rampas alternativas. Esses obstáculos podem ser a causa de transtornos que prejudicam o deslocamento pela cidade, inclusive a dirigibilidade de veículos particulares ou do transporte público ${ }^{28}$.

Os pontos fortes deste estudo são o pioneirismo na caracterização do ambiente alimentar no entorno das residências de famílias beneficiárias do PBF. A identificação da existência dos estabelecimentos e a sua agregação são importantes na elaboração e avaliação de políticas públicas já existentes que visam a melhoria de acesso e disponibilidade de alimentos saudáveis, sobretudo para a população em questão, exposta a piores condições sociais, econômicas e ambientais de saúde.

Quanto às limitações do presente estudo, pondera-se que foram utilizados dados secundários não validados, que podem ter dados faltantes em relação aos estabelecimentos que comercializam alimentos e às famílias beneficiárias do PBF. Outro ponto importante a ser destacado é a temporalidade dos dados: as informações das famílias e dos estabelecimentos são de 2014, mas o último Censo realizado foi em 2010.

Uma crise econômica de dimensão global afetou o Brasil, e consequentemente os estados e municípios, sendo agravada pela crise política e econômica vivenciada em 2015, que aumentou o desemprego e reduziu as taxas de crescimento do país. Além disso, o arroxo das políticas neoliberais, amparado pela reforma da previdência, constitui a mais agressiva proposta de restrições de direitos sociais da história da seguridade social no Brasil ${ }^{58}$. Todo esse contexto pode ter mudado drasticamente os determinantes sociais econômicos e ambientais da saúde, revelando um cenário pior do que o encontrado neste estudo ${ }^{58}$. Apesar disso, os resultados são relevantes, por mostrarem mais um cenário desfavorável ao qual os beneficiários do PBF estão expostos.

Outra limitação é a falta de informações sobre o mercado informal no banco de dados utilizado para a coleta de informação dos estabelecimentos. Contudo, o mercado informal normalmente é representado por estabelecimentos de venda predominante de alimentos ultraprocessados, o que provavelmente não mudaria a característica geral do ambiente alimentar urbano de Ouro Preto. Além disso, a utilização da distância euclidiana é uma limitação, pois ela não retrata o caminho real a ser percorrido entre a residência e o estabelecimento, e também não considera a dificuldade de acesso aos locais, uma vez que não avalia outras variáveis que impactam direta ou indiretamente nesse acesso (meio de transporte, segurança, declives, entre outros). Apesar disso, as informações de distância obtidas já refletem a potencial dificuldade de acesso dos beneficiários do PBF, principalmente aos estabelecimentos de venda de alimentos saudáveis, podendo ser na realidade muito maior.

Para modificar essa exposição desigual aos estabelecimentos que comercializam alimentos, que afeta sobretudo a população usuária do PBF, são necessárias políticas públicas inovadoras, intersetoriais e efetivas que contemplem a multidimensionalidade da SAN e do ambiente alimentar comunitário e atendam de fato às regiões $\mathrm{e}$ às populações menos privilegiadas. A cidade de Belo Horizonte (MG) possui diversos exemplos de políticas públicas viáveis que visam aumentar o acesso físico e econômico aos alimentos in natura e minimamente processados, como as feiras-livres, as feiras orgânicas, o direto da roça, os mercados municipais e os sacolões municipais abastecer ${ }^{55}$. A adoção dessas políticas pode melhorar o ambiente alimentar no entorno das residências das famílias beneficiárias, caso esses equipamentos sejam instalados em regiões onde há maior aglomeração dessas famílias. 


\section{Colaboradores}

ML Araújo e LL Rocha redigiram o artigo, revisaram criticamente e aprovaram a versão a ser publicada. GB Silva e MC Pessoa participaram da concepção e desenho da pesquisa, da análise e interpretação dos dados, da redação e da revisão crítica, bem como aprovaram a versão a ser publicada. TG Novaes, CAM Lima e LL Mendes participaram da concepção e desenho da pesquisa, da análise e interpretação dos dados e aprovaram a versão a ser publicada.

\section{Financiamento}

O presente trabalho foi realizado com apoio da Coordenação de Aperfeiçoamento de Pessoal de Nível Superior (CAPES) - Código de Financiamento 001 .

\section{Referências}

1. Casemiro JP, Valla VV, Guimarães BL. Direito humano à alimentação adequada: um olhar urbano. Cien Saude Colet 2010;15(4):2085-2093.

2. Story M, Kaphingst KM, Brien RRO, Glanz K. Creating healthy food and eating environments: policy and environmental approaches. Annu Rev Public Health 2008; 29:253-272.

3. Moore LV, Diez Roux AV, Nettleton JA, Jacobs Jr DR. Associations of the local food environment with diet quality--a comparison of assessments based on surveys and geographic information systems: the multi -ethnic study of atherosclerosis. Am J Epidemiol 2008; 167(8):917-924.

4. Zenk SN, Schulz AJ, Kannan S, Lachance LL, Mentz G, Ridella W. Neighborhood retail food environment and fruit and vegetable intake in a multiethnic urban population. Am J Heath Promot 2009; 23(4):255-264.

5. Glanz K, Sallis JF, Saelens BE, Frank LD. Healthy nutrition environments: concepts and measures. Am J Health Promot 2005; 19(5):330-333.

6. White M. Food access and obesity. Obes Rev 2007; 8(Suppl. 1):99-107.

7. Jetter KM, Cassady DL. The Availability and cost of healthier food alternatives. Am J Prev Med 2006; 30(1):38-44.

8. Walker RE, Keane CR, Burke JG. Disparities and access to healthy food in the United States: a review of food deserts literature. Health Place 2010; 16(5):876884 .

9. Breyer B, Voss-Andreae A. Food mirages: geographic and economic barriers to healthful food access in Portland, Oregon. Health Place 2013; 24:131-139.

10. National Collaborating Centre for Environmental Health (NCCEH). Food deserts and food swamps: a primer. Vancouver: NCCEH; 2017.

11. Ministério da Saúde (MS). Secretaria de Atenção à Saúde. Manual de orientações sobre o Programa Bolsa Família. Brasília: MS; 2009.

12. Brasil. Lei no 11.346 , de 15 de setembro de 2006. Cria o Sistema Nacional de Segurança Alimentar e Nutricional - SISAN com vistas em assegurar o direito humano à alimentação adequada e dá outras providências. Diário Oficial da União 2006; 15 set.

13. Lima FEL, Rabitoll EI, Dias MRMG. Estado nutricional de população adulta beneficiária do Programa Bolsa Família no município de Curitiba PR. Rev Bras Epidemiol 2011; 14(2):198-206.

14. Attanasio O, Gómez LC, Heredia P, Vera-Hernandez M. The short-term impact of a conditional cash subsidy on child health and nutrition in Colombia. Institute for Fiscal Studies; 2005.

15. Testa MG, Fronza P, Petrini M, Prates JC. Análise da contribuição do Programa Bolsa Família para o enfrentamento da pobreza. Rev Adm Publica 2013; 47(6):1519-1541.

16. Burlandy L. Transferência condicionada de renda e segurança alimentar e nutricional. Cien Saude Colet 2007; 12(6):1441-1451.

17. Cotta RMM, Machado JC. Programa Bolsa Família e segurança alimentar e nutricional no Brasil: revisão crítica da literatura. Rev Panam Salud Publica 2013; 33(1)54-60. 
18. Oliveira JS, Lira PIC, Maia SR, Sequeira LAS, Amorim RCA, Batista Filho M. Insegurança alimentar e estado nutricional de crianças de Gameleira, zona da mata do nordeste brasileiro. Rev Bra Saude Mater Infant 2010; 10(2):237-245.

19. Faria AA, Silva RBD. Direito à alimentação, transferência de renda e progressividade. Revista Jurídica da Presidência 2016; 18(114):145-168.

20. Fernald LC, Gertler PJ, Hou X. Cash component of conditional cash transfer program is associated with higher body mass index and blood pressure in adults. J Nutr 2008; 138(11):2250-2257.

21. Monteiro F, Schimidt ST. O Programa Bolsa Família no contexto da segurança alimentar e nutricional no Brasil. Saude Transform Soc 2014; 5(3):91-97.

22. Ricardo CZ, Claro RM. Custo da alimentação e densidade energética da dieta no Brasil, 2008-2009. Cad Saude Publica 2012; 28(12):2349-2361.

23. Baptistella JCF. Avaliação de programas sociais: uma análise do impacto do Bolsa Família sobre o consumo de alimentos [Dissertação]. Sorocaba: Universidade Federal de São Carlos; 2012.

24. Cabral MJ, Vieira KA, Sawaya AL, Florêncio TMMT. Perfil socioeconômico, nutricional e de ingestão alimentar de beneficiários do Programa Bolsa Família. Estud Av 2013; 27(78).

25. Santos LN, Carneiro DF, Schimidt ST, Bezerra I. Estratégias de acesso aos alimentos pelas famílias titulares de direito do Programa Bolsa Família.Divers@ Revista Eletrônica Interdisciplinar 2015; 8(2):84-94.

26. Lignani JB, Sichieri R, Burlandy L, Salles-Costa R. Changes in food consumption among the Programa Bolsa Família participant families in Brazil. Public Health Nutr 2011; 14(5):785-792.

27. Instituto Brasileiro de Geografia e Estatística (IBGE). Ouro Preto [Internet]. [acessado 2019 Dez 18]. Disponível em: https://cidades.ibge.gov.br/brasil/mg/ouro-preto/panorama

28. Nogueira JAN. Estudo diagnóstico do desenvolvimento urbano e do sistema de transporte da cidade de Ouro Preto/MG: subsídios para a implantação de um teleférico [Dissertação]. Ouro Preto: Universidade Federal de Ouro Preto; 2014.

29. Instituto Brasileiro de Geografia e Estatística (IBGE). Comissão Nacional de Classificação. Classificação Nacional de Atividades Econômicas 2011 [Internet]. [acessado 2016 Set 12]. Disponível em: http://concla. ibge.gov.br/classificacoes/por-tema/atividades-economicas/classificacao-nacional-de-atividades-economicas.html/.

30. Monteiro CA, Cannon G, Levy RB, Moubarac JC, Jaime P, Martins AP, Canella Daniela, Louzada Maria, Parra Diana. NOVA. The star shines bright. World Nutrition 2016; 7(1-3);28-38.

31. Câmara Interministerial de Segurança Alimentar e Nutricional. Estudo técnico mapeamento dos desertos alimentares no Brasil. Brasiília: Secretaria-Executiva da Câmara Interministerial de Segurança Alimentar e Nutricional Ministério do Desenvolvimento Social; 2018.

32. Stanton RA. Food Retailers and Obesity. Curr Obes Rep 2015; 4(1):54-59.
33. Larsen K, Cook B, Stone MR. Food access and children's BMI in Toronto, Ontario: assessing how the food environment relates to overweight and obesity. Int J Public Health 2015; 60(1):69-77.

34. Instituto Brasileiro de Geografia e Estatística (IBGE). Censo 2010 [Internet]. [acessado 2017 Mar 2]. Disponível em: http://www.censo2010.ibge.gov.br

35. Instituto Brasileiro de Geografia e Estatística (IBGE). Resolução $n^{\circ} 1$, de 25 de fevereiro de 2005. Altera a caracterização do Sistema Geodésico Brasileiro. Brasília: IBGE; 2005.

36. Pessoa MC, Mendes LL, Gomes CS, Martins PA, Velasquez-Melendez G. Food environment and fruit and vegetable intake in a urban population: a multilevel analysis. BMC Public Health 2015; 15:1012.

37. Jaime PC, Duran AC, Sarti FM, Lock K. Investigating environmental determinants of diet, physical activity, and overweight among adults in Sao Paulo, Brazil. Journal of Urban Health 2011; 88(3):567-581.

38. Passos CM, Maia EG, Levy RB, Martins APB, Claro RM. Association between the price of ultra-processed foods and obesity in Brazil. Nutr Metab Cardiovasc Dis 2020; 30(4):589-598.

39. Maia EG, Passos CM, Levy RB, Martins APB, Mais LA, Claro RM. What to expect from the price of healthy and unhealthy foods over time? The case from Brazil. Public Health Nutr 2020; 23(4):579-588.

40. Machado PP, Claro RM, Canella DS, Sarti FM, Levy RB. Price and convenience: the influence of supermarkets on consumption of ultra-processed foods and beverages in Brazil. Appetite 2017; 116:381-388.

41. Araujo ML, Nascimento DR, Lopes MS, Passos CM, Lopes ACS. Condições de vida de famílias brasileiras: estimativa da insegurança alimentar. $R$ Bras Est Pop 2020; 37:1-17.

42. Oliveira SP, Muniz LB, Marliere CA, Freitas SN, Cancho VG, Freitas FV, Fonseca KZ, Carvalho LR. Características do comércio de alimentos no Município de Ouro Preto (Minas Gerais): subsídios para a segurança alimentar. Cadernos de Debate 2003:1-27.

43. Correa EN, Padez CMP, Abreu AH, Vasconcelos FAG. Geographic and socioeconomic distribution of food vendors: a case study of a municipality in the Southern Brazil. Cad Saude Publica 2017; 33(2):e00145015

44. Matozinhos FP. Fatores ambientais associados à obesidade [Tese]. Belo Horizonte: Universidade Federal de Minas Gerais; 2015.

45. Moore LV, Diez Roux AV. Associations of neighborhood characteristics with the location and type of food stores. Am J Public Health 2006; 96(2):325331.

46. Larson NI, Story MT, Nelson MC. Neighborhood environments: disparities in access to healthy foods in the U.S. Am J Prev Med 2009; 36(1):74-81.

47. Engler-Stringer R, Shah T, Bell S, Muhajarine N. Geographic access to healthy and unhealthy food sources for children in neighbourhoods and from elementary schools in a mid-sized Canadian city. Spat Spatio-temporal Epidemiol 2014; 11:23-32.

48. Suglia SF, Shelton RC, Hsiao A, Wang YC, Rundle A, Link BG. Why the neighborhood social environment is critical in obesity prevention. J Urban Health 2016; 93(1):206-212. 
49. Oliveira SP, Muniz LB, Marliere CA, Freitas SN, Fonseca KZ, Carvalho LR, Cancho VG. Hábitos de compra de alimentos da população de Ouro Preto (Minas Gerais). Segurança Alimentar e Nutricional 2005; 12(1):1-9.

50. Instituto Brasileiro de Análises Sociais e Econômicas. Repercussões do Programa Bolsa Família na segurança alimentar e nutricional das famílias beneficiadas (2007-2008). Brasília: Instituto Brasileiro de Análises Sociais e Econômicas; 2008.

51. Conselho Nacional de Segurança Alimentar e Nutricional. Cartilha da Lei de Segurança Alimentar e Nutricional. Brasília: Consea; 2006.

52. Ministério de Desenvolvimento Social e Combate à Fome (MDS). O Estado de Segurança Alimentar e Nutricional no Brasil. Um retrato multidimensional. Brasília: MDS; 2014

53. Food and Agriculture Organization of the United Nations. The State of Food Insecurity in the World. Meeting the 2015 international hunger targets: taking stock of uneven progress. Rome: Food and Agriculture Organization; 2015.

54. Araújo ML, Mendonça RD, Lopes Filho JD, Lopes AC. Association between food insecurity and food intake. Nutrition 2018; 54:54-59.

55. Menezes MC, Lopes ACS, Araújo ML. O ambiente alimentar e o acesso a frutas e hortaliças: "Uma metrópole em perspectiva”. Saúde Soc 2017; 26(3):764-773.

56. Ransley JK, Donnelly JK, Botham H, Khara TN, Greenwood DC, Cade JE. Use of supermarket receipts to estimate energy and fat content of food purchased by lean and overweight families. Appetite 2003; 41(2):141-8.

57. Cooksey-Stowers K, Schwartz MB, Brownell KD. Food Swamps Predict Obesity Rates Better Than Food Deserts in the United States. Int J Environ Res Public Health 2017; 14(11):1366.

58. Silva MLL. Contrarreforma da Previdência Social sob o comando do capital financeiro. Serv Soc Soc 2018; 131:130-54.

Artigo apresentado em 30/06/2020

Aprovado em 07/01/2021

Versão final apresentada em 09/01/2021

Editores-chefes: Romeu Gomes, Antônio Augusto Moura da Silva 
